\title{
A Cascading Failures Perspective Based Mesoscopic Reliability Model of Weighted Public Transit Network considering Congestion Effect and User Equilibrium Evacuation
}

\author{
Lin Zhang, ${ }^{1,2,3}$ Jian Lu $\mathbb{D},{ }^{1,2,3}$ Man Long, ${ }^{1,2,3}$ Shu-bin Li $\mathbb{D}^{4},{ }^{4}$ and Jialin Zhou $\mathbb{D}^{5}$ \\ ${ }^{1}$ Jiangsu Key Laboratory of Urban ITS, Southeast University, Nanjing 210096, China \\ ${ }^{2}$ Jiangsu Province Collaborative Innovation Center of Modern Urban Traffic Technologies, Southeast University, Nanjing 210096, China \\ ${ }^{3}$ School of Transportation, Southeast University, Nanjing 210096, China \\ ${ }^{4}$ Department of Traffic Management Engineering, Shandong Police College, Jinan 250014, China \\ ${ }^{5}$ Griffith School of Engineering, Griffith University, 58 Parklands Dr., Southport, QLD 4215, Australia
}

Correspondence should be addressed to Jian Lu; lujian_1972@seu.edu.cn

Received 12 January 2018; Accepted 1 April 2018; Published 10 May 2018

Academic Editor: Mauro Gaggero

Copyright (c) 2018 Lin Zhang et al. This is an open access article distributed under the Creative Commons Attribution License, which permits unrestricted use, distribution, and reproduction in any medium, provided the original work is properly cited.

\begin{abstract}
To establish the optimal route layout estimation technology based on reliability optimization, a more accurate and realistic reliability model of a weighted public transit network (PTN) is the indispensable basis. This paper establishes a cascading failures (CFs) perspective based mesoscopic reliability model for measuring PTN survivability. First, a modeling method for abstracting weighted PTN and determining its initial passenger flow and bearing capacity is proposed, making the network passenger flow pattern follow the aggregated flow pattern. Second, three basic concepts (time step, congestion effect of a road section, and CFs judging method) for establishing the CFs model are defined to clarify the overall evolution process of CFs. Furthermore, the aggregated passenger flow evacuation that exists in an emergency occurring at a station (i.e., failure load dynamic redistribution (FLDR)) is considered as a conscious dynamic game process through following the user equilibrium rule. As a result, a novel CFs model that considers congestion effect and user equilibrium evacuation is obtained. Finally, based on the collected data of Jinan's PTN, a case simulation analysis is conducted to verify the adaptability of this model through showing a significantly different dynamics characteristic with the existing FLDR patterns and to provide optimization direction for effectively controlling PTN survivability, that is, guide the transformation among varying FLDR patterns through some technical measures or traffic policies.
\end{abstract}

\section{Introduction}

A new tendency for alleviating traffic congestion, parking problem, and car pollution from the mesoscopic or macroscopic perspective of traffic network is becoming a consensus of both traffic scholars and traffic engineers, that is, emerging traffic network related researches [1-6]. Continuing this tendency, a combined transportation mode dominated by "public transit plus nonmotorized mode (e.g., public bicycle)" is considered as the optimal trip mode, that is, transforming the traditional mode into a green and lowcarbon system. "Public transit plus nonmotorized mode (e.g., public bicycle)" can be regarded as "core trip plus last mile trip." Because public transit network (PTN) is the core mode of this combined mode, its reliability plays an important role in ensuring the effective use of public transit system for alleviating urban traffic congestion and is widely concerned by scholars. Many studies were conducted from the perspective of service reliability, including the headway reliability $[7,8]$ and the running time reliability [9-12]. However, few researches have focused on the mesoscopic reliability and the system reliability, that is, PTN survivability. PTN survivability originates from complex network theory, and it can be classified as static survivability and dynamic 
survivability (i.e., CFs) by recognizing whether the failure station or connected edge contributes to the failure of adjacent stations or connected edges. Since 1990s, Watts and Strogatz [13] proposed the small-world network in Nature journal, and Barabasi and Albert [14] subsequently modeled the scale-free network in Science journal; then Albert et al. [15] stated their work on survivability of complex network in Nature journal. Additionally, it should be noted that the CFs are important factors affecting the network reliability and are common in most real complex networks, including cluster supply networks [16], power grids [17-19], social networks [20], and transportation networks [21]. These pioneering works form a trend to study basic theory such as the complex network and its survivability. In particular, a PTN is a typical complex network; thus it follows the above development process.

1.1. Literature Review. Empirical studies have shown that the PTN is a unified system formed by stations, routes, and their interrelationships. A PTN contains extensive dynamic passenger flow information, making it a large, complex, and dynamic system. In other words, a PTN is a typical complex network [22-24], so applying complex network theory on it can reveal the interaction between network topology and function. Following the above development process of complex network survivability, the researches on PTN survivability are divided into the static survivability and the CFs perspective based dynamic survivability.

Regarding the PTN static survivability, Wu et al. [25] measured the network efficiency by minimum transfer frequency; then they found that, because of the influences of large clustering coefficient, the local network efficiency of Beijing's PTN is greatly influenced by the deliberate attacks. Taking the PTNs of major cities all over the world as the examples, Berche et al. [26] analyzed the PTN reliability dynamics under various attack modes. By the combined use of complex network theory and GIS technology, Yang et al. [27] simulated the subway system to deal with the destruction of random attacks and deliberate attacks. Then, they found that the scale-free characteristics of subway system make it possess good survivability when dealing with random attacks rather than deliberate attacks. Through proposing a novel betweenness importance based network structure entropy, Fu et al. [28] developed a new method to measure the PTN static survivability. This method reveals the interaction mechanism among PTN survivability, station heterogeneity, and network structure entropy.

Regarding the CFs perspective based PTN dynamic survivability, by simultaneously analyzing the static survivability and dynamic survivability of Foshan's PTN, Zou et al. [29] found that the key stations can be determined through comparing the similarities and differences between the two types of survivability. Zhang et al. [30] developed the CFs model of Jinan's weighted PTN under single station happening emergency, and, based upon this model, they found that the intensity size of failure station, the station load tolerance parameter, and the station load distribution control parameter have important influences on cascading survivability. He et al. [31] proposed a path navigation

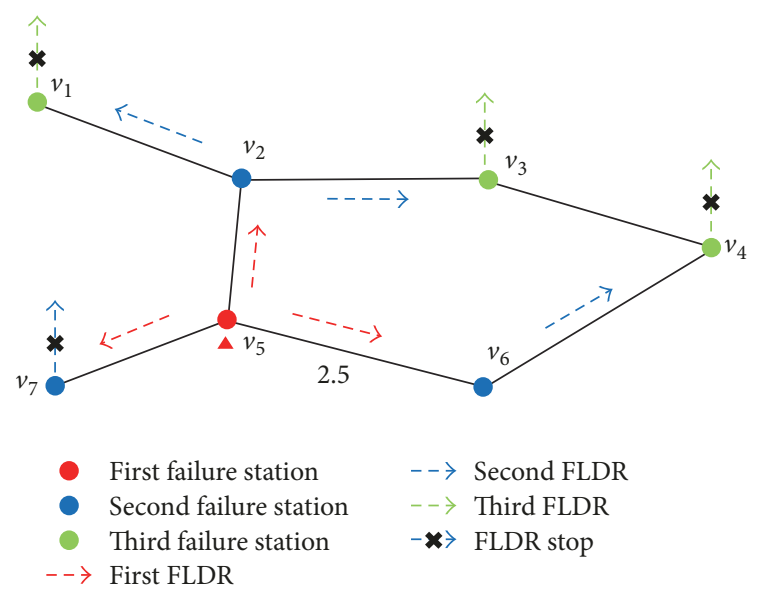

FIgure 1: The CFs and FLDR in a weighted PTN.

strategy that describes the traffic flow spread of PTN and established an improved PTN CFs model. This improved path navigation strategy could make the node load definition value be consistent with the actual flow value. Song et al. [32] designed the key part recognition method of a PTN considering the failure probability and failure consequence; then they further proposed the calculation method of station importance.

To sum up, PTN survivability is an interdisciplinary study of transportation engineering and system science, which has aroused widespread interests among scholars. The above studies have deepened the understanding of PTN reliability from multiple perspectives, especially the CFs perspective based PTN dynamic survivability, which are described in the follows:

(i) What are the CFs and the failure load dynamic redistribution (FLDR) in a weighted PTN?

When an emergency occurs at a key station (i.e., station failure), the passenger flow transited through this station will be evacuated to adjacent stations (i.e., FLDR), which may cause the adjacent stations to exceed their capacity, thus extending the evacuation process. The above process gradually expands outward from the original failure station and may ultimately cause all of the stations in the PTN to fail, as shown in Figure 1. In particular, this process is described based on an important assumption; that is, the passenger flow volumes transited through these failure stations are strong enough to cause their adjacent stations to fail. However, some adjacent stations will fail and other adjacent stations will not fail; that is, not all adjacent stations fail under the practical circumstance.

1.2. Objectives and Contributions. In this interdisciplinary study, the ultimate objective is to get an accurate and realistic description of the CFs model. It should be noted that previous studies on the PTN CFs essentially focused on network physical topology, although they used the actual networks. This limitation is that it causes CFs to lack the necessary description from the perspective of transportation 
engineering, which inspires our objectives and contributions to this field.

Reference [33] demonstrated that the origin-destination (OD) path selection behavior of the user (vehicle) is a conscious dynamic game process. Under mesoscopic perspective (CFs are described into a mesoscopic phenomenon in largescale network), the evacuation of bus passenger flow during an emergency is based on a situational assumption because the passenger evacuation (i.e., FLDR) is essentially a type of OD path selection behavior. Additionally, this CFs based mesoscopic reliability model is established under a passenger flow system with aggregated transit pattern; the passenger evacuation is conducted through the vehicle aggregated transit pattern, which does not consider passenger individual behaviors. In other words, there is no such phenomenon where passengers walk from failure station to other stations and only the phenomenon where buses have to change operating routes to skip failure station because the CFs happen under emergencies exists. It simultaneously explains why this cascading failures perspective based reliability model is considered as the mesoscopic reliability model. Thus, an FLDR based on the proportion of adjacent station capacity [30, 34, 35] or on the proportion of coupled capacity [36], which both lack descriptions of the driver (user) path selection behavior and road congestion effect, does not conform to the actual operation of a PTN.

The contributions of this paper mainly contain three aspects:

(i) One contribution in the aspect of methodological advances is that a CFs perspective based mesoscopic reliability model of a weighted PTN considering congestion effect and user equilibrium evacuation is established to provide a novel perspective for precisely understanding the FLDR triggered by emergencies. This mesoscopic model keeps a balance between description precision and calculation efficiency, making it widely applied in different cities in the future.

(ii) One contribution in the aspect of practical application advances is that if a more accurate and realistic reliability model of a weighted PTN is found, it can make a contribution to establishing the optimal route layout estimation technology based on reliability optimization. In other words, this kind of extensive related studies will provide a novel way to plan PTN under the perspective with optimal reliability.

(iii) The other contribution in the aspect of practical application advances is that it provides the optimization direction for effectively controlling PTN survivability, that is, guide the transformation among varying FLDR patterns through some technical measures or traffic policies, so as to obtain the optimal cascading survivability under a certain $\lambda$ (characterizing the size of the station bearing capacity).

More specifically, a modeling method for establishing weighted PTN and determining its initial passenger flow and bearing capacity is proposed, making the network passenger flow pattern follow the aggregated flow pattern. Second,

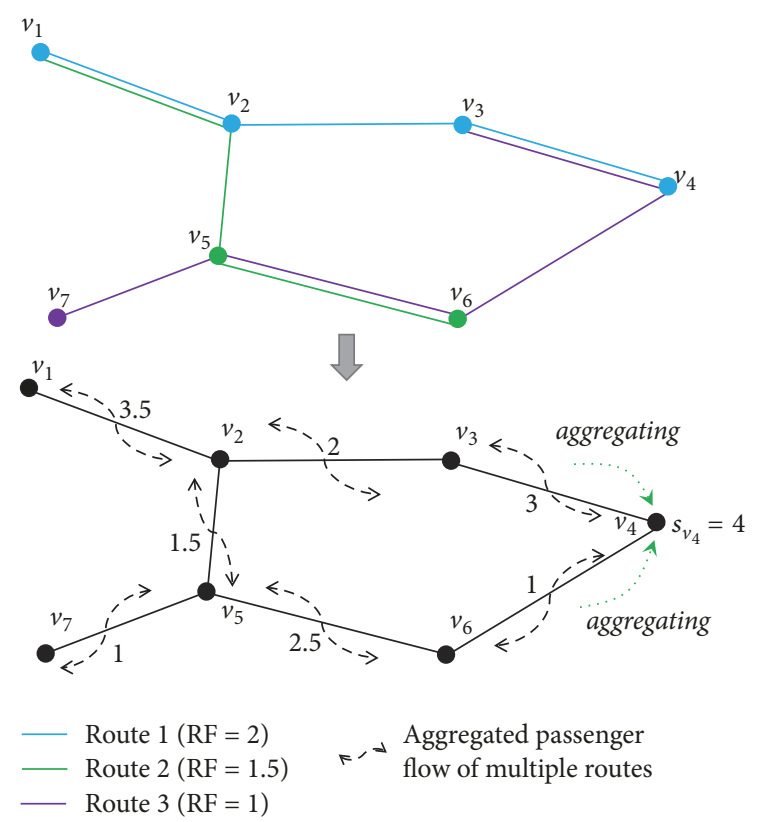

FIGURE 2: Modeling process of a weighted PTN.

three basic concepts (time step, congestion effect of a road section, and CFs judging method) for establishing the CFs model are defined to clarify the overall evolution process of CFs. Furthermore, the aggregated passenger flow evacuation that exists in an emergency occurring at a station (i.e., FLDR) is considered as a conscious dynamic game process through following the user equilibrium rule. As a result, a novel CFs model that considers congestion effect and user equilibrium evacuation is obtained. Finally, based on the collected data of Jinan's PTN, a case simulation analysis is conducted to verify the adaptability of this model through showing a significantly different dynamics characteristic with the existing FLDR patterns and to provide optimization direction for effectively controlling PTN survivability, that is, guide the transformation among varying FLDR patterns through some technical measures or traffic policies.

The rest of this paper is organized as follows: Section 2 provides the modeling method and mechanism of a weighted PTN. The modeling process of the CFs model for a weighted PTN is presented in Section 3. The case study of Jinan's PTN is presented in Section 4. Finally, Section 5 contains the summaries and prospects.

\section{Modeling Method of a Weighted PTN}

2.1. Modeling Process. This paper establishes the weighted PTN by using the modified space $L$ method, which describes the passenger flow carried by the PTN based on the aggregation method [30,36]. More specifically, if one bus route sequentially passes through two adjacent stations, then there is a connected edge between the two stations. Then, the edge weights are relative values of the bus route density and the departure frequency of the two stations. Through a simple example shown in Figure 2, the modeling process of this 
method is presented. Route 1 consists of stations $v_{1}, v_{2}, v_{3}$, and $v_{4}$, and its relative frequency (expressed as RF) is equal to 2; route 2 consists of stations $v_{1}, v_{2}, v_{5}$, and $v_{6}$, and RF $=1.5$; and route 3 consists of stations $v_{7}, v_{5}, v_{6}, v_{4}$, and $v_{3}$, and RF $=$ 1 .

A weighted PTN $G$ consists of the node set $V$, the edge set $E$, and the weighted adjacency matrix $A_{L}$, as shown in (1). The weighted adjacency matrix $A_{L}$ is calculated following this example: $\bar{e}_{v_{1} v_{2}}$ is the multiple edge between stations $v_{1}$ and $v_{2}$, and its weight is calculated as $1 \times \mathrm{RF}^{\text {route1 }}+1 \times \mathrm{RF}^{\text {route2 }}=$ $2+1.5=3.5$; that is, $w_{\bar{e}_{v_{1}^{\prime} v_{2}^{\prime}}}=3.5$ and $a_{12}=3.5$. Additionally, no edge exists between stations $v_{1}$ and $v_{3}$, so $a_{13}=\infty$.

$$
\begin{aligned}
& G=\left(V, E, A_{L}\right) \\
& \left\{\begin{aligned}
V & =\left\{v_{1}, v_{2}, v_{3}, v_{4}, v_{5}, v_{6}, v_{7}\right\} \\
E= & \left\{\bar{e}_{v_{1} v_{2}}, e_{v_{2} v_{3}}, \bar{e}_{v_{3} v_{4}}, e_{v_{4} v_{6}}, \bar{e}_{v_{5} v_{6}}, e_{v_{5} v_{7}}, e_{v_{2} v_{5}}\right\} \\
A_{L}= & {\left[\begin{array}{ccccccc}
0 & 3.5 & \infty & \infty & \infty & \infty & \infty \\
3.5 & 0 & 2 & \infty & 1.5 & \infty & \infty \\
\infty & 2 & 0 & 3 & \infty & \infty & \infty \\
\infty & \infty & 3 & 0 & \infty & 1 & \infty \\
\infty & 1.5 & \infty & \infty & 0 & 2.5 & 1 \\
\infty & \infty & \infty & 1 & 2.5 & 0 & \infty \\
\infty & \infty & \infty & \infty & 1 & \infty & 0
\end{array}\right] }
\end{aligned}\right.
\end{aligned}
$$

2.2. Determining Passenger Flow Pattern. Continuing the above modeling concept, the stations are regarded as the generating sources of passenger flow, and the edges (road sections) are regarded as the carriers for achieving passenger flow transit between adjacent stations. Thus, the initial passenger flow and bearing capacity of network can be defined based on the stations.

The initial station passenger flow (load) and bearing capacity (capacity) were defined in our previous study [36]. More specifically, the station intensity $s_{v_{i}}$ is the most important topological property of the weighted PTN, which is defined as the sum of weights of the connected edges directly connected to station $v_{i}$. The stations with larger intensity correspond to the stations with higher route density and larger passenger flow volume. For example, the intensity of station $v_{4}$ in Figure 2 is obtained by aggregating $a_{34}=3$ and $a_{64}=1$; that is, $s_{v_{4}}$ is the sum of the 4 th row or 4 th column of weighted adjacency matrix $A_{L}$. Reference [36] showed that the station load not only is closely related to its key topological properties but also has a certain linear relationship with the number of adjacent stations. The larger the number of adjacent stations is, the more frequent the load transits between stations will be. Thus, the initial load $L_{v_{i}}$ and capacity $C_{v_{i}}$ of station $v_{i}$ are defined as follows:

$$
\begin{aligned}
L_{v_{i}} & =\left[s_{v_{i}}^{\alpha}\left(\sum_{\Gamma_{v_{i}}} s_{v_{j}}\right)^{1-\alpha}\right]^{\beta}, \\
C_{v_{i}} & =(1+\lambda) L_{v_{i}},
\end{aligned}
$$

where $\Gamma_{v_{i}}$ denotes the adjacent station set of station $v_{i}, v_{j} \in \Gamma_{v_{i}}$; $s_{v_{i}}$ denotes the intensity of station $v_{i}$; and $s_{v_{j}}$ denotes the intensity of station $v_{j}$; $\alpha$ denotes the load weight adjustment parameter $(\alpha \in[0,1])$, which is negatively correlated with the impact of the adjacent stations, and it can actively adjust the impact weight of the adjacent stations when the station load is defined; $\beta$ denotes the control parameter of the load distribution $(\beta \geq 1)$, which is positively correlated with the heterogeneity of PTN, and its variation has a certain internal relationship with the future CFs evolution of a PTN; and $\lambda$ denotes the station load tolerance parameter, which characterizes the size of the station bearing capacity.

Therefore, a station can be in one of two states, that is, the normal state or the failure state. Combining the actual operation characteristics of a PTN, the states of station $v_{i}$ (expressed as $\mathrm{SS}_{v_{i}}$ ) are divided into the normal state (expressed as $\mathrm{SS}_{v_{i}}^{\text {normal }}$ ) and failure state (expressed as $\mathrm{SS}_{v_{i}}^{\text {failure }}$ ), shown as follows:

$$
\mathrm{SS}_{v_{i}}= \begin{cases}\mathrm{SS}_{v_{i}}^{\text {normal }}, & \text { if } L_{v_{i}} \leq C_{v_{i}} \\ \mathrm{SS}_{v_{i}}^{\text {failure }}, & \text { if } L_{v_{i}}>C_{v_{i}}\end{cases}
$$

The above processes determine the initial passenger flow and bearing capacity of a weighted PTN, and the passenger flow patterns determined using the above method follow the aggregated flow pattern. In particular, the passenger flow does not exist in the form of a route transit but rather exists in the form of road section transit under the aggregated flow pattern. In other words, the multiple routes running between two stations are treated as a unified system without a common route problem. Additionally, because of the special modeling process, the established passenger flow system is essentially the average passenger flow system of certain period of time, which does not consider the traffic time-varying conditions.

\section{CFs Model of a Weighted PTN}

\subsection{Three Basic Concepts}

3.1.1. Time Step. A special time scale for dynamically modeling CFs needs to be introduced; that is, the CFs process of a PTN is divided into different time steps in accordance with the time order, and the time step is expressed as $l: l=$ $1,2,3 \cdots n$. Time step $l$ is the discrete time scale that describes the dynamic update of the station load. Therefore, the CFs model is described as the nonlinear dynamic system with time-space discretization under this time scale.

In particular, time step $l$ does not represent a moment of time but rather represents a certain length of time that is sufficiently long to complete load transit between two stations under CFs conditions, as shown in Figure 3. Each time step consists of three time points, that is, the beginning point, middle point, and end point. For example, time step $l=n$ consists of the beginning of $l=n$, the middle of $l=n$, and the end of $l=n$.

3.1.2. Congestion Effect of a Road Section. The user equilibrium distribution of an urban traffic network originates from 


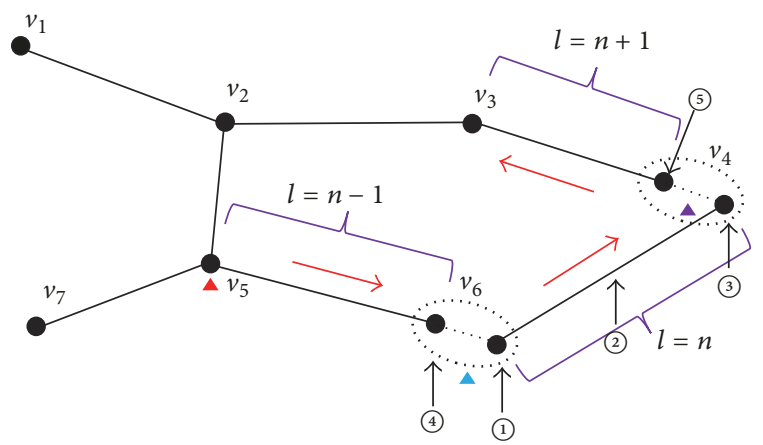
(1) The beginning of $l=n$
(2) The middle of $l=n$
- First failure
(3) The end of $l=n$
(4) The end of $l=n-1$
- Second failure
- Third failure
(5) The beginning of $l=n+1$

FIGURE 3: Evolution principle of the proposed time step.

the dependence of the road section (edge) impedance on traffic flow. The road section impedance is also known as the congestion effect of a road section, but most previous works ignore its existence when describing FLDR. Because it is measured using the impedance function, this paper proposes a modified impedance function based on the American Bureau of Public Roads function (BPR function) and the topological characteristics of a weighted PTN. More specifically, the impedance $w_{v_{i} v_{j}, l=n}$ of road section $e_{v_{i} v_{j}}$ in $l=n$ is defined as follows:

$$
w_{v_{i} v_{j}, l=n}=w_{v_{i} v_{j}}^{0}\left(1+a\left(\frac{x_{v_{i} v_{j}, l=n}}{\mathrm{Cp}_{v_{i} v_{j}}}\right)^{b}\right),
$$

where the parameters $a=0.15$ and $b=4$ are typically used; $x_{v_{i} v_{j}, l=n}$ denotes the flow of road section $e_{v_{i} v_{j}}$ in $l=n$; and $w_{v_{i} v_{j}}^{0}$ (zero-flow impedance) denotes the travel time on road section $e_{v_{i} v_{j}}$ when there is no flow and is closely related to the betweenness of the road sections, as shown in (8). C $\mathrm{p}_{v_{i} v_{j}}$ denotes the connected edge transit capacity of road section $e_{v_{i} v_{j}}$ and has no relationship with $l$. Analogously referring to [37], the connected edge transit capacity is defined as follows, which characterizes the road section capacity of public transit:

$$
\begin{aligned}
w_{v_{i} v_{j}}^{0} & =B_{v_{i} v_{j}}^{\tau}, \\
\mathrm{Cp}_{v_{i} v_{j}} & =\left(s_{v_{i}} \cdot s_{v_{j}}\right)^{\theta},
\end{aligned}
$$

where $B_{v_{i} v_{j}}$ denotes the betweenness of road section $e_{v_{i} v_{j}}$ (edge betweenness), that is, the number of shortest paths through road section $e_{v_{i} v_{j}}$, and it is calculated based on the geospatial PTN. More specifically, the geospatial PTN is established by using space $L$ method $[30,36]$, and it is closely related to the urban road network, particularly to the urban road network in which the PTN operates. $\tau$ denotes the control parameter of zero-flow impedance of the road section, which is positively correlated with the heterogeneity of zeroflow impedance of the road section, and it can actively control the uniformity of the network initial impedance distribution. $\theta$ denotes the control parameter of the connected edge transit capacity, which is positively correlated with the road section's ability to transit the passenger flow of a certain traffic mode. In other words, the parameter $\theta$ can effectively control the capacity of the road section between two adjacent stations, according to the key topological characteristics. follows:

Therefore, the modified impedance function is defined as

$$
w_{v_{i} v_{j}, l=n}=w_{v_{i} v_{j}}^{0}\left(1+a\left(\frac{x_{v_{i} v_{j}, l=n}}{\left(s_{v_{i}} \cdot s_{v_{j}}\right)^{\theta}}\right)^{b}\right) .
$$

Because $B_{v_{i} v_{j}}$ is the road section betweenness of the special urban road network in which the PTN operates, $w_{v_{i} v_{j}}^{0}$ considers the influences of public transit mode, private car mode, and other traffic modes. By adding $w_{v_{i} v_{j}}^{0}$ to (6), the modified impedance function $w_{v_{i} v_{j}, l=n}^{\text {lower }}$ also considers the influences of these traffic modes; that is, the proposed characterization method of congestion effect has considered the influences of public transit mode, private car mode, and other traffic modes on the urban road.

\subsubsection{CFs Judging Method}

(i) Station Cascade State. Clarify the difference between the station cascade state proposed in this subsection and the station state defined in Section 2. The CFs happening to the station must meet two basic conditions: the station is in the failure state (determined by (3)) and has an adjacent station set. If a station does not have effective adjacent stations, then its load cannot be transited in the network; that is, the FLDR does not exist and this part of load is completely paralyzed. Accordingly, the station cascade state is defined to characterize this circumstance. More specifically, station $v_{i}$ fails in $l=n\left(v_{j} \in \Gamma_{v_{i}, l=n}\right)$; then, whether the network continues the CFs in $l=n+1$ is determined by the state transition function $f_{v_{j}}$ of station $v_{j}$ (this paper specifies that station $v_{i}$ fails earlier than station $v_{j}$, and station $v_{j}$ is adjacent to station $\left.v_{i}\left(v_{j} \in \Gamma_{v_{i}, l=n}\right)\right)$, shown as follows:

$$
\begin{aligned}
f_{v_{j}}: \mathrm{CSS}_{v_{j}, l=n+1} & =f\left(\operatorname{SS}_{v_{j}, l=n+1}, \Gamma_{v_{j}, l=n+1}\right) \\
& =\mathrm{CSS}_{v_{j}, l=n+1}^{\text {failure }} \vee \mathrm{CSS}_{v_{j}, l=n+1}^{\text {normal }},
\end{aligned}
$$

where $\operatorname{CSS}_{v_{j}, l=n+1}$ denotes the cascade state of station $v_{j}$ in $l=$ $n+1$, which can be further divided into the state of continuing CFs (expressed as $\operatorname{CSS}_{v_{j}, l=n+1}^{\text {failure }}$ ) and that of terminating CFs (expressed as $\operatorname{CSS}_{v_{j}, l=n+1}^{\text {normal }}$ ).

Figure 4 shows mechanism of state transition function. Stations $v_{6}$ and $v_{7}$ simultaneously fail at the beginning of $l=2$; that is, stations $v_{6}$ and $v_{7}$ are in failure states $\mathrm{SS}_{v_{6}, l=2}^{\text {failure }}$ and $\mathrm{SS}_{v_{7}, l=2}^{\text {failure }}$, respectively, whereas station $v_{2}$ is in normal state 


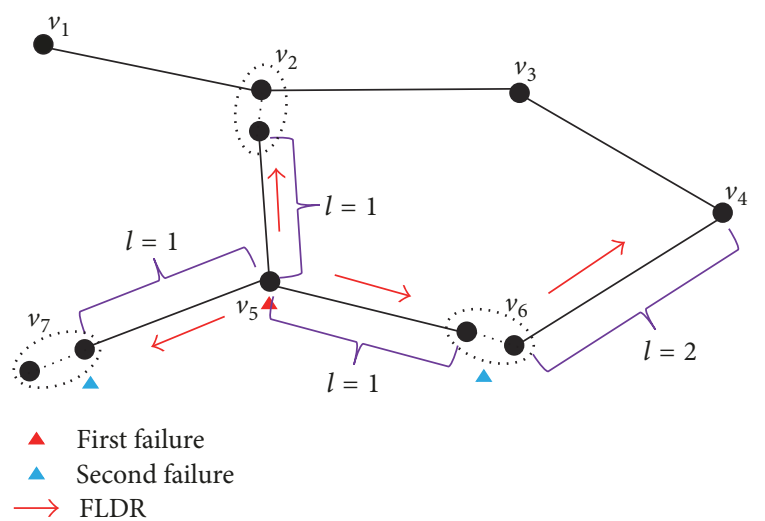

FIGURE 4: Mechanism of state transition function $f_{v_{j}}$.

$\mathrm{SS}_{v_{2}, l=2}^{\text {normal }}$. For example, for station $v_{6}$ to be in the cascade state $\mathrm{CSS}_{v_{6}, l=2}^{\text {failure }}$, it must simultaneously be in the failure state $\left(\mathrm{SS}_{v_{6}, l=2}^{\mathrm{failure}}\right)$ and have an adjacent station set $\left(\Gamma_{v_{6}, l=2} \neq \varnothing\right)$; otherwise, it is in the cascade state $\operatorname{CSS}_{v_{6}, l=2}^{\text {normal }}$. Therefore, $f_{v_{2}}$, $f_{v_{6}}$, and $f_{v_{7}}$ are shown as follows:

$$
\begin{array}{r}
f_{v_{2}}: f\left(\mathrm{SS}_{v_{2}, l=2}^{\text {normal }}, \forall \Gamma_{v_{2}, l=2}\right) \\
f_{v_{6}}: f\left(\mathrm{SS}_{v_{6}, l=2}^{\text {failure }}, \Gamma_{v_{6}, l=2} \neq \varnothing\right) \Longrightarrow \mathrm{CSS}_{v_{2}, l=2}^{\text {normal }}, \\
f_{v_{7}}: f\left(\mathrm{SS}_{v_{7}, l=2}^{\text {failure }}, \Gamma_{v_{7}, l=2}=\varnothing\right) \Longrightarrow \mathrm{CSS}_{v_{6}, l=2}^{\text {failure }},
\end{array}
$$

(ii) Load Updating Rule. Taking station $v_{6}$ in Figure 4 as an example, the load updating rule of CFs model is illustrated. More specifically, station $v_{6}$ receives the redistributed load from station $v_{5}$ at the end of $l=1$; then its load is updated at the beginning of $l=2$. Therefore, formulate the above process as follows:

$$
\begin{gathered}
v_{6, l=1}: \text { receive } \Delta L_{v_{5} \rightarrow v_{6}, l=1}, \\
v_{6, l=2} \text { : update } L_{v_{6}, l=2}=L_{v_{6}, l=1}+\Delta L_{v_{5} \rightarrow v_{6}, l=1},
\end{gathered}
$$

where $\Delta L_{v_{5} \rightarrow v_{6}, l=1}$ denotes the redistributed load transited from failure station $v_{5}$ to the adjacent station $v_{6}$ at the middle of $l=1$. This load can be determined using the user equilibrium assignment algorithm under the CFs, which is proposed in Section 3.3.

\section{(iii) Judging Criteria}

(a) Continuing CFs. If the cascade state of station $v_{j}$ is $\mathrm{CSS}_{v_{j}, l=n+1}^{\text {failure }}$ at the beginning of $l=n+1$, then the PTN will continue CFs, as shown in (12). In other words, station $v_{j}$ continues the CFs when it is simultaneously in the failure state $\mathrm{SS}_{v_{j}, l=n+1}^{\text {failure }}$ and has an adjacent station set (i.e., $\Gamma_{v_{j}, l=n+1} \neq \varnothing$ ).

$$
\mathrm{CSS}_{v_{j}, l=n+1}^{\text {failure }}=f\left(\mathrm{SS}_{v_{j}, l=n+1}^{\text {failure }}, \Gamma_{v_{j}, l=n+1} \neq \varnothing\right) \text {. }
$$

(b) Terminating CFs. If the cascade state of station $v_{j}$ is $\mathrm{CSS}_{v_{j}, l=n+1}^{\text {normal }}$ at the beginning of $l=n+1$, then the PTN will
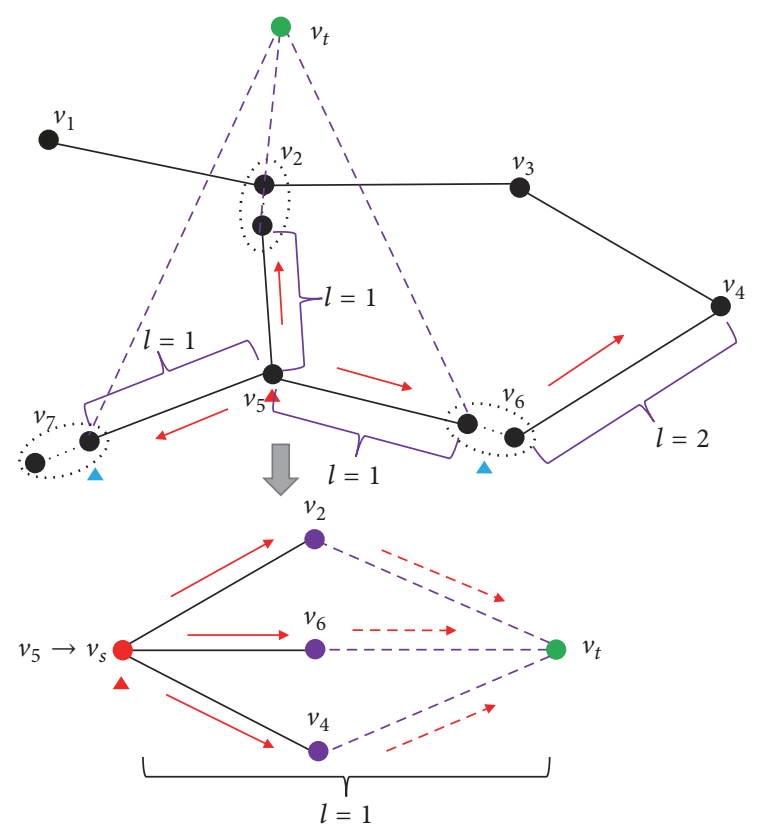

$\begin{aligned} \Delta & \text { First failure } \\ \longrightarrow & \text { Second failure } \\ \longrightarrow & \text { FLDR }\end{aligned}$

FIgURE 5: Method for transforming FLDR into a general user equilibrium problem.

terminate the CFs, as shown in (13). In other words, station $v_{j}$ terminates the CFs when it is not in the failure state or does not have an adjacent station set.

$$
\begin{aligned}
\mathrm{CSS}_{v_{j}, l=n+1}^{\text {normal }}= & f\left(\mathrm{SS}_{v_{j}, l=n+1}^{\text {normal }}, \forall \Gamma_{v_{j}, l=n+1}\right) \\
& \vee f\left(\forall \mathrm{SS}_{v_{j}, l=n+1}, \Gamma_{v_{j}, l=n+1}=\varnothing\right) .
\end{aligned}
$$

3.2. Overall Evolution Process of CFs. The overall evolution process of CFs can be divided into plenty of stages in terms of the order of the time steps. More specifically, the failure station is graded by the time step when it fails, that is, the $n$th grade failure stations are the stations that fail in $l=n$, which can be expressed as the failure station set $\Phi_{l=n}$. Furthermore, the overall evolution process of FLDR is described through the simple example in Figure 5. In this figure, station $v_{5}$ fails at the beginning of $l=1$, and its load $L_{v_{5}, l=1}$ is redistributed to the adjacent station set $\Gamma_{v_{5}, l=1}=\left\{v_{2}, v_{6}, v_{7}\right\}$ following certain rules (such as FLDR following user equilibrium). The redistributed load is transited at the middle of $l=1$ and the adjacent stations receive it at the end of $l=1$. Then, $L_{\Gamma_{v_{5}, l=1}}=\left\{L_{v_{2}, l=2}, L_{v_{6}, l=2}, L_{v_{7}, l=2}\right\}$ is updated at the beginning of $l=2$; that is, $L_{v_{2}, l=2}=L_{v_{2}, l=1}+\Delta L_{v_{5} \rightarrow v_{2}, l=1}, L_{v_{6}, l=2}=L_{v_{6}, l=1}+$ $\Delta L_{v_{5} \rightarrow v_{6}, l=1}$, and $L_{v_{7}, l=2} \stackrel{L_{v_{7}}, l=1}{=}+\Delta L_{v_{5} \rightarrow v_{7}, l=1}$, respectively. At this moment, stations $v_{6}$ and $v_{7}$ are determined by judging criteria to continue the CFs but that station $v_{2}$ is determined to terminate the CFs. Therefore, at the middle of $l=2$, $L_{v_{6}, l=2}$ is redistributed to $\Gamma_{v_{6}, l=2}=\left\{v_{4}\right\}$ according to (9), while $L_{v_{2}, l=2}$ and $L_{v_{7}, l=2}$ are not redistributed according to (8) and 
(10), respectively. Finally, $L_{\Gamma_{v_{6}, l=3}}=\left\{L_{v_{4}, l=3}\right\}$ is updated at the beginning of $l=3$ (i.e., $L_{v_{4}, l=3}=L_{v_{4}, l=2}+\Delta L_{v_{6} \rightarrow v_{4}, l=2}$ ).

In particular, the following should be noted:

(i) The CFs model is proposed as a mesoscopic reliability model; thus not all microscopic individual behaviors are taken into consideration. In other words, the CFs model keeps a balance between description precision and calculation efficiency, which is proven to be advantageous in application through extensive empirical studies such as cluster supply networks [16], power grids [17-19], and public transit network [36, 38].

(ii) This mesoscopic reliability model is established under a passenger flow system with aggregated transit pattern; the passenger evacuation (i.e., FLDR) is conducted through the vehicle aggregated transit pattern, which does not consider individual behaviors. In other words, there is no such phenomenon where passengers walk from failure station to other stations. Additionally, the buses have to change operating routes to skip failure station because the CFs happen under emergencies.

\subsection{FLDR following User Equilibrium}

3.3.1. User Equilibrium Model under CFs. Wardrop's first principle is also known as the user equilibrium problem. In this learning process, the road network will reach the equilibrium under the circumstance that users have a clear understanding of the road network and try to choose the shortest route. Therefore, all used paths have equal and minimized travel time, and no user may decrease his travel time through unilateral action [39]. The user equilibrium problem is to find the edge flows that satisfy the user equilibrium rule when all the origin-destination entries have been appropriately assigned. To better understand this principle, Beckmann et al. [40] transformed the user equilibrium problem into an equivalent mathematical programming model, which effectively describes the user equilibrium problem, that is, the user equilibrium model.

Due to the edges' existing congestion effect which is usually ignored, this paper considers the FLDR to be a conscious dynamic game process through following the user equilibrium rule. The adopted user equilibrium model is used for capturing changes in the route choice behavior of the users in the case of a failure in the network. This route choice behavior is the cumulative result of individual decisions made by rational users who tend to minimize the generalized cost of their own trips based on their previous experiences in the network. However, this behavior may change when a failure occurs in the network because private car users and bus drivers cannot accurately estimate the travel times in the network under this condition. Additionally, although stochastic user equilibrium models are expected to provide better approximations of the route choice behavior of unexperienced users when a failure occurs in reality, the FLDR is still considered to follow the user equilibrium rule because these two models are similar when the network is crowded [33]. Indeed, the network is crowded when CFs occur, and the user equilibrium model can be better applied in a large-scale network. Therefore, we assume that all users have perfect perception on the impedances of different road sections based on the Advanced Traffic Information System (ATIS) and that the network state condition is relatively steady.

Analogously referring to the convex combination method proposed by Sheffi [33] for solving user equilibrium problem and the actual PTN operation characteristics, this paper proposes a method for transforming FLDR into a general user equilibrium problem under a single time step $(\Delta l=1)$, as shown in Figure 5. For example, station $v_{5}$ fails at the beginning of $l=1$, and its load $L_{v_{5}, l=1}$ is redistributed to the adjacent station set $\Gamma_{v_{5}, l=1}=\left\{v_{2}, v_{6}, v_{7}\right\}$ following the user equilibrium rule, that is, the FLDR that follows user equilibrium evacuation.

The steps are as follows.

Step 1. Failure station $v_{5}$ is considered the starting point $v_{s}$ of the OD pair $\left\langle v_{s} v_{t}\right\rangle$.

Step 2. The adjacent station set $\Gamma_{v_{5}, l=1}=\left\{v_{2}, v_{6}, v_{7}\right\}$ is considered the ending point $v_{t}$ of the OD pair $\left\langle v_{s} v_{t}\right\rangle$, and stations $v_{2}, v_{6}$, and $v_{7}$ are connected to $v_{t}$ through the dummy edges (purple dotted edges $e_{v_{2} v_{t}}, e_{v_{6} v_{t}}$, and $e_{v_{7} v_{t}}$ in Figure 5), which are different paths between the OD pair $\left\langle v_{s} v_{t}\right\rangle$.

Step 3. The three paths of the OD pair $\left\langle v_{s} v_{t}\right\rangle$ are $\left\{e_{v_{s} v_{2}}, e_{v_{2} v_{t}}\right\}$, $\left\{e_{v_{s} v_{6}}, e_{v_{6} v_{t}}\right\}$, and $\left\{e_{v_{s} v_{7}}, e_{v_{7} v_{t}}\right\}$.

Step 4. The impedances of the dummy edges are set equal to each other, so that their influences on the congestion effect can be neglected. Thus, the congestion effect on the paths $\left(\left\{e_{v_{s} v_{2}}, e_{v_{2} v_{t}}\right\},\left\{e_{v_{s} v_{6}}, e_{v_{6} v_{t}}\right\}\right.$, and $\left.\left\{e_{v_{s} v_{7}}, e_{v_{7} v_{t}}\right\}\right)$ is essentially characterized using the impedances of $w_{v_{s} v_{2}, l=1}, w_{v_{s} v_{6}, l=1}$, and $w_{v_{s} v_{7}, l=1}$.

Step 5. The failure load $L_{v_{5}, l=1}$ is considered the total travel volume $q_{v_{s} v_{t}, l=n}$ of the OD pair $\left\langle v_{s} v_{t}\right\rangle$.

Step 6. The FLDR between failure station $v_{5}$ and its adjacent station set $\Gamma_{v_{5}, l=1}$ is transformed into the user equilibrium distribution of the total travel volume $L_{v_{5}, l=1}$ on paths $\left\{e_{v_{s} v_{2}}, e_{v_{2} v_{t}}\right\},\left\{e_{v_{s} v_{6}}, e_{v_{6} v_{t}}\right\}$, and $\left\{e_{v_{s} v_{7}}, e_{v_{7} v_{t}}\right\}$.

Thus, the user equilibrium model under the CFs is defined as follows:

$$
\begin{array}{ll}
\min _{\Gamma_{v_{i}, l=n}} & Z(x)=\sum_{e_{v_{i} v_{j}}} \int_{0}^{x_{v_{i} v_{j}, l=n}} w_{v_{i} v_{j}}(x) d x \\
\text { s.t. } & \sum_{k} f_{k, l=n}^{v_{s} v_{t}}=q_{v_{s} v_{t}, l=n}=L_{v_{s}, l=n}, \quad \forall v_{s}, v_{t} \\
& f_{k, l=n}^{v_{s} v_{t}} \geq 0, \quad \forall v_{s}, v_{t}, k \\
& x_{v_{i} v_{j}, l=n}=\sum_{v_{s}} \sum_{v_{t}} \sum_{k} f_{k, l=n}^{v_{s} v_{t}} \delta_{v_{i} v_{j}, k, l=n}^{v_{s} v_{t}}, \quad \forall v_{i}, v_{j},
\end{array}
$$

where $\Gamma_{v_{i}, l=n}$ denotes the adjacent station set of station $v_{i}$ in $l=n ; v_{j} \in \Gamma_{v_{i}, l=n} ; w_{v_{i} v_{j}}(\cdot)$ denotes the impedance function of 
road section $e_{v_{i} v_{j}}$, which uses the BPR function given in (4); $f_{k, l=n}^{v_{s} v_{t}}$ denotes the passenger flow of the $k$ th path connecting to the OD pair $\left(v_{s} v_{t}\right)$ in $l=n$; and $q_{v_{s} v_{t}, l=n}$ denotes the total travel volume of the OD pair $\left(v_{s} v_{t}\right)$ in $l=n$, that is, the load $L_{v_{s} l=n}$ of failure station $v_{s}$. Equation (15) is the conservation relation between all path passenger flows and the OD total travel volume. Equation (16) ensures that all path passenger flows are positive; and (17) is the association relationship between the road section passenger flow and the path passenger flow. Specifically, $\delta_{v_{i} v_{j}, k, l=n}^{v_{s} v_{t}}=1$ when road section $e_{v_{i} v_{j}}$ is the $k$ th path of the OD pair $\left(v_{s} v_{t}\right)$ in $l=n$; otherwise, $\delta_{v_{i} v_{j}, k, l=n}^{v_{s} v_{t}}=0$.

3.3.2. User Equilibrium Assignment Algorithm under the CFs. The Frank-Wolfe algorithm, a kind of convex combination algorithm, was originally used to solve quadratic programming problems with linear constraints [41] and was later used to solve the user equilibrium model [42]. This method is emphasized in this paper for its effectiveness in determining the equilibrium flows for transportation networks [43]. Based on the Frank-Wolfe algorithm and the user equilibrium model under the $\mathrm{CFs}$, the user equilibrium assignment algorithm under a single time step $(\Delta l=1)$ is proposed, shown as follows.

Step 0 (initializing).

Step 0.1. Grade the failure stations. Each station of the weighted PTN is given a unique number $v_{1}, v_{2}, \ldots, v_{n}$, and the stations that fail at the beginning of $l=n$ are expressed as the failure station set $\Phi_{l=n}$, that is, the $n$th grade failure stations.

Step 0.2. Determine the OD pair of FLDR and the zero-flow impedance. During this time step, a new station is randomly selected from set $\Phi_{l=n}$, which contains multiple adjacent road sections (i.e., adjacent road section set $\left\{e_{v_{i}} v_{j} \mid v_{j} \in \Gamma_{v_{i}, l=n}\right\}$, such as $j=2,3,4$ in Figure 5) connected to the adjacent station set $\Gamma_{v_{i}, l=n}$. Furthermore, let the impedance for each adjacent road section be the zero-flow impedance; that is, $w_{v_{i} v_{j}, l=n}=w_{v_{i} v_{j}}^{0}$.

Step 0.3. Load the initial redistribution load. Based on the "all-or-nothing" method, the road section with the minimum impedance in the adjacent road section set $\left\{e_{v_{i} v_{j}} \mid v_{j} \in \Gamma_{v_{i}, l=n}\right\}$ will load the OD total travel volume $L_{v_{i} l=n}$, thus obtaining the adjacent road section flow set $\left\{x_{v_{i} v_{j}, l=n} \mid v_{j} \in \Gamma_{v_{i}, l=n}\right\}$. Then, set counter $h=1$.

Step 1 (updating the impedance of the road section). For all adjacent road sections $e_{v_{i} v_{j}}$, let

$$
w_{v_{i} v_{j}, l=n}^{h}=w_{v_{i} v_{j}}\left(x_{v_{i} v_{j}, l=n}^{h}\right) .
$$

Step 2 (determining the search direction). According to the updated $\left\{w_{v_{i} v_{j}, l=n}^{h} \mid v_{j} \in \Gamma_{v_{i} l=n}\right\}, L_{v_{i} l=n}$ is loaded onto the road section that has the minimum impedance based on the "all-or-nothing" method, thus obtaining the auxiliary road section flow set $\left\{y_{v_{i} v_{j}, l=n}^{h} \mid v_{j} \in \Gamma_{v_{i}, l=n}\right\}$.
Step 3 (determining the iterative step size using linear search). To solve the one-dimensional minimal problem,

$$
\min _{\varsigma \in[0,1]} \sum_{e_{v_{i} v_{j}}} \int_{0}^{x_{v_{i} j_{j}, l=n}^{h}+\zeta\left(y_{v_{i} v_{j}, l=n}^{h}-x_{v_{i} v_{j}, l=n}^{h}\right)} w_{v_{i} v_{j}}(x) d x,
$$

let its solution be $\varsigma_{n}$, that is, the iterative step size.

Step 4 (updating the flow of the road section). For all adjacent road sections $e_{v_{i} v_{j}}$, let

$$
x_{v_{i} v_{j}, l=n}^{h+1}=x_{v_{i} v_{j}, l=n}^{h}+c_{n}\left(y_{v_{i} v_{j}, l=n}^{h}-x_{v_{i} v_{j}, l=n}^{h}\right) .
$$

Step 5 (judging convergence). If it satisfies

$$
\frac{\sqrt{\sum_{e_{v_{i} v_{j}}}\left(x_{v_{i} v_{j} l}^{h+1}, x_{v_{i}}^{h}-x_{v_{i} v_{j} l=n}^{h}\right)^{2}}}{\sum_{e_{v_{i} v_{j}}} x_{v_{i} v_{j}, l=n}^{h}} \leq \varepsilon,
$$

then the FLDR of this failure station stops iterating, and the algorithm goes to Step 6; otherwise, set $h=h+1$ and go to Step 1 ( $\varepsilon$ is a smaller number, and its general value is 0.0001 ).

Step 6 (ending the judgment of a single time step). The algorithm ends when all stations in set $\Phi_{l=n}$ are selected. In particular, the flow set $\left\{x_{v_{i} v_{j}, l=n}^{h+1} \mid v_{j} \in \Gamma_{v_{i}, l=n}\right\}$ is the user equilibrium flow pattern for FLDR in $l=n$.

\subsection{Measurement Indicator}

3.4.1. Ratio of Station CFs. The number of initial failure stations is $h$; then their loads are redistributed to adjacent stations; when PTN terminates CFs, the number of stations that ultimately fail is $M$. Therefore, the ratio of station CFs (expressed as RCF) is defined as follows:

$$
\mathrm{RCF}=\frac{M}{h(N-h)}=\frac{\sum_{l} \sum_{v_{i}} N_{\mathrm{SS}_{v_{i} l=n}^{\text {fallure }}}}{h(N-h)},
$$

where $N$ denotes the total number of stations in PTN; $\sum_{v_{i}} N_{\mathrm{SS}_{v_{i}, l}=n}$ denotes the number of failure stations in $l=n$.

The smaller RCF is under a certain parameter combination, the fewer failure stations there will be, demonstrating a larger cascading survivability.

3.4.2. Global and Local Ratios of CFs in a Time Step. The number of CFs in a time step (expressed as $\mathrm{TCF}_{l=n}: \mathrm{TCF}_{l=n}=$ $\left.\sum_{v_{i}} N_{\mathrm{SS}_{v_{i}=n}^{\text {fallure }}}\right)$ was proposed in previous work, which can intuitively describe the complex dynamic evolution process of CFs [36]. It should be noted that this measurement indicator can measure the overall CFs evolution process but cannot effectively describe the local strength of the CFs. Therefore, $\mathrm{TCF}_{l=n}$ is extended to the global ratio of the CFs in a time step (expressed as $\mathrm{RTCF}_{\text {global }, l=n}$ ) and the local ratio of CFs in a time step (expressed as $\mathrm{RTCF}_{\text {local }, l=n}$ ), which can be defined as follows:

$$
\begin{aligned}
\mathrm{RTCF}_{\text {global }, l=n} & =\frac{\mathrm{TCF}_{l=n}}{N}=\frac{\sum_{v_{i}} N_{\mathrm{SS}_{v_{i}} \text { falluen }}}{N}, \\
\mathrm{RTCF}_{\text {local }, l=n} & =\frac{\mathrm{TCF}_{l=n}}{N_{\Gamma_{\Phi_{l=n-1}}}}=\frac{\sum_{v_{i}} N_{\mathrm{SS}_{v_{i}, l=n}}^{\text {failure }}}{N_{\Gamma_{\Phi_{l=n-1}}}},
\end{aligned}
$$


where $\Gamma_{\Phi_{l=n-1}}$ denotes the adjacent station set of all stations contained in set $\Phi_{l=n-1} ; N_{\Gamma_{\Phi_{l=n-1}}}$ denotes the number of stations of set $\Gamma_{\Phi_{l=n-1}}$. RTCF $_{\text {global }, l=n}$ can be used to intuitively observe the CFs strength of each time step, and the time step with the largest CFs strength is the best time to optimize and control cascading survivability. $\mathrm{RTCF}_{\text {local }, l=n}$ can effectively describe the CFs local strength of each time step.

To sum up, this paper provides a full viewing perspective for better understanding CFs, that is, the aggregated and discretized perspectives (i.e., RCF and $\mathrm{RTCF}_{\text {global }, l=n}-$ $\mathrm{RTCF}_{\text {local } l=n}$ ) and the global and local perspectives (i.e., $\mathrm{RTCF}_{\text {global }, l=n}$ and $\left.\mathrm{RTCF}_{\text {local }, l=n}\right)$.

\section{Case Simulation Study}

4.1. Data Description and Simulation Initialization. Jinan city is one of the top ten congested cities in China [43] and its balance between supply and demand of traffic is extremely fragile. To alleviate the urban traffic congestion and improve the efficiency of limited road resource utilization, a welldeveloped ground public transit system has been established. The case study encompasses 195 bus routes and 1433 bus stations and the processed relative departure frequency of each bus route from Jinan in May 2014, which are taken as the sample data [44]. This study data is open to all researchers by visiting the website of Jinan public transit company (i.e., "http://www.jnbus.com.cn"), but it needs collecting and subsequently processing the data by ourselves because the data is in the form of public transit schedules. An important discovery, which is that Jinan's PTN is a typical complex network, has been obtained by analyzing the detailed topology characteristics in our previous work, that is, [28, 36, 45]. Different from the conventional research based on the WattsStrogatz (WS) small-world and Barabási-Albert (BA) scalefree network models $[13,14]$, verifying the adaptability of a CFs model based on an actual PTN has more practical significance. Thus, based on the proposed modeling method of a weighted PTN, Jinan's weighted PTN is established.

Furthermore, the proposed CFs perspective based mesoscopic reliability model is simulated based on a self-designed MATLAB program. Its adaptability is verified by showing a significantly different dynamics characteristic with the existing CFs models for measuring PTN survivability. More specifically, the dynamics characteristic of the proposed FLDR that follows user equilibrium evacuation (expressed as FLDR 1) is compared with the dynamics characteristics of the FLDR that follows average evacuation [46] (expressed as FLDR 2; i.e., the load of failure station is averagely distributed to its adjacent stations.) and the FLDR based on the proportion of adjacent station capacity [30,34] (expressed as FLDR 3).

For improving the sensitivity of simulation experiment, a high-sensitivity control parameters combination of the proposed CFs model needs to be selected as the basic comparison parameter combination. The basic comparison parameter combination is also called the experience value combination, which can be obtained using actual data from Jinan's PTN and a typical test method. More specifically, the experience value of parameter $\alpha$ is 0.7 , the experience value of

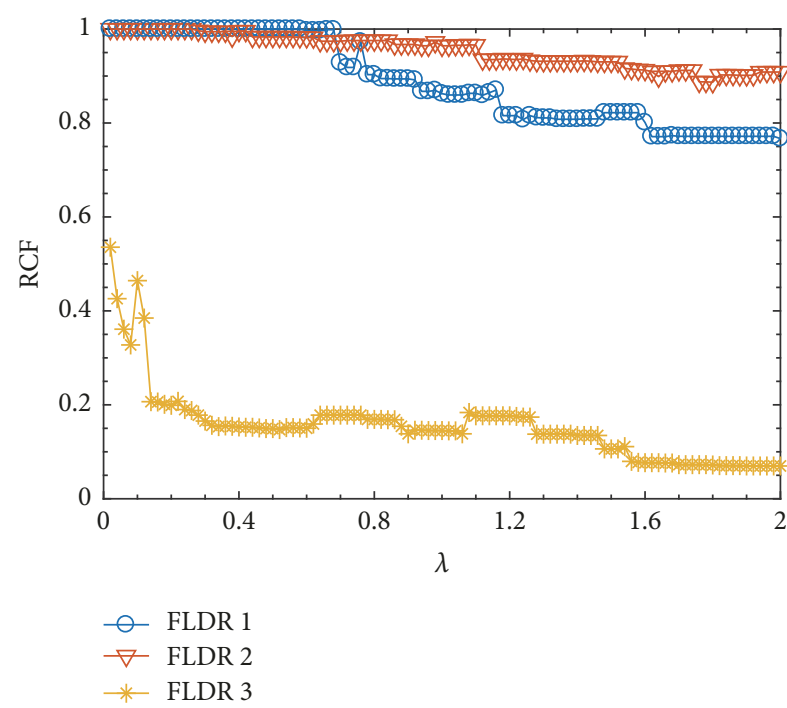

FIGURE 6: Simulation verification analysis of RCF evolution under varying FLDR rules.

parameter $\beta$ is 6.5 , the experience value of parameter $\tau$ is 0.8 , and the experience value of parameter $\theta$ is 1.1 . Additionally, the simulation experiment focuses on the station with the maximum load which failed when $\lambda \in[0,2]$, and the simulation step size is $\lambda=0.02$.

4.2. Simulation Analysis. The simulation experiments were performed on a Dell machine equipped with Windows $10 \times 64$ operating system and an Intel Core i7-7700HQ processor (CPU $2.8 \mathrm{GHz} \times 4$ ), RAM $8 \mathrm{~GB}$. Under this computer simulation environment, we operate three selfdesigned MATLAB programs for three FLDR patterns, which are all operated in the state of MATLAB program starting parallel pool connected to 4 workers. Thus, the simulation time consumptions of FLDR 1, FLDR 2, and FLDR 3 are, respectively, $547.473 \mathrm{~s}, 308.735 \mathrm{~s}$, and $252.187 \mathrm{~s}$, preliminarily demonstrating that the dynamics evolutions of three FLDR patterns are significantly different.

The smaller the RCF is under the parameter combination ( $\alpha=0.7, \beta=6.5, \tau=0.8$, and $\theta=1.1$ ), the fewer the failure stations there will be, demonstrating larger cascading survivability. Figure 6 shows the dynamic evolution of RCF under varying FLDR rules. This figure approximately follows $\mathrm{RCF}^{\mathrm{FLDR} 2}>\mathrm{RCF}^{\mathrm{FLDR} 1}>\mathrm{RCF}^{\mathrm{FLDR} 3}$, indicating that different FLDRs drive a quite huge difference of dynamic evolution characteristics. This difference validates the need for studying the FLDR pattern. Due to the network used for simulating being an actual weighted PTN, we think that FLDR 1 measures and captures the CFs more realistically based on the two following reasons:

(i) The congestion effect that existed in road section where the buses and other traffic modes are running has been agreed by extensive traffic scholars and engineers $[33,37]$, and this effect will have a significant impact on the FLDR. 


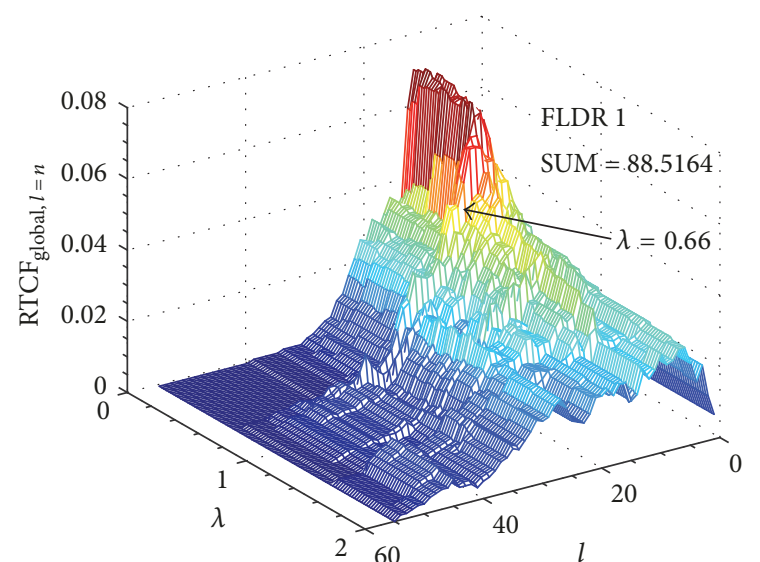

(a)

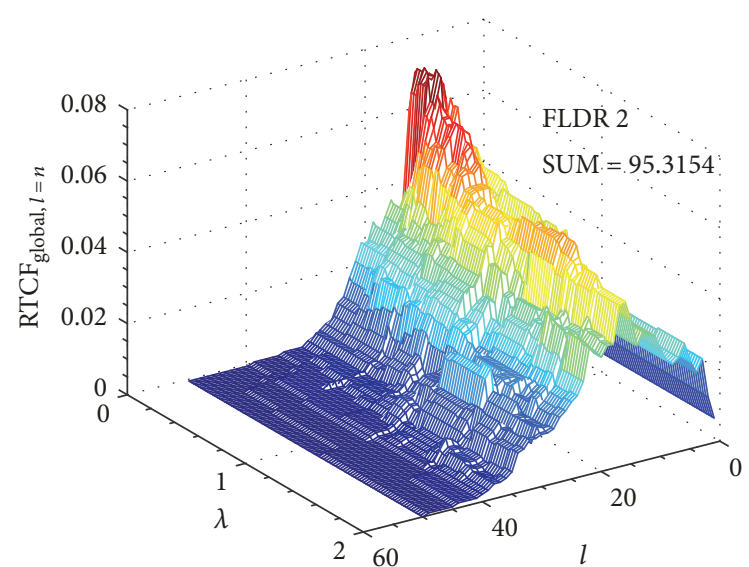

(b)

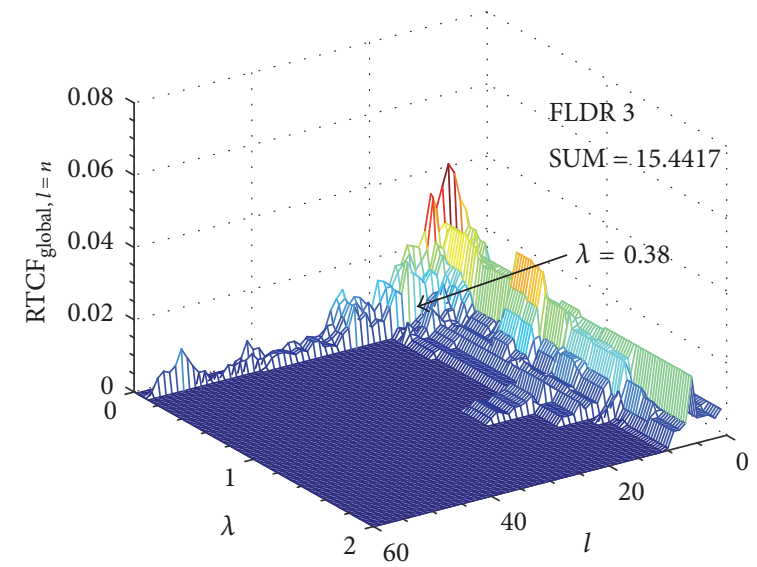

(c)

FIGURE 7: Simulation verification analysis of $\mathrm{RTCF}_{\text {global }, l=n}$ evolution under varying FLDR rules.

(ii) Different from the abstract theoretical network model and the power grids, the dynamic evolution of transportation network is affected by the drivers and passengers, that is, the various human behaviors. Thus, the FLDR following user equilibrium evacuation is a more realistic pattern, which is essentially a conscious dynamic game process.

Additionally, it should be noted that Figure 6 also provides optimization direction for effectively controlling PTN survivability. For example, this paper considers that FLDR 1 is more close to the realistic situation, so we can control the FLDR by observing which FLDR pattern has the optimal cascading survivability under certain $\lambda$ (characterizing the size of the station bearing capacity).

$\mathrm{RTCF}_{\text {global }, l=n}$ and RCF have the same measurement effect under the overall perspective of CFs evolution. Comparatively, $\mathrm{RTCF}_{\text {global, } l=n}$ can be used to intuitively observe the CFs strength of each time step, and the time step with the largest CFs strength is the best time to optimize and control cascading survivability. Figure 7 shows the dynamic evolution of $\mathrm{RTCF}_{\text {global }, l=n}$ under varying FLDR rules, which more intuitively verifies the conclusions obtained from Figure 6. Additionally, by observing Figure 7(c), it indicates that the
FLDR based on the proportion of adjacent station capacity is the optimal pattern with the largest cascading survivability. This interesting phenomenon points out the direction that we can make efforts, that is, guide FLDR 1 to be transformed into FLDR 3 through some technical measures or traffic policies.

$\mathrm{RTCF}_{\text {local }, l=n}$ can effectively describe the local strength of the CFs. More specifically, the larger $\mathrm{RTCF}_{\text {local }, l=n}$ is under a certain parameter combination, the larger the local strength of the CFs will be in this time step, demonstrating smaller local cascading survivability. Figure 8 shows the dynamic evolution of $\mathrm{RTCF}_{\text {local }, l=n}$ under varying FLDR rules, which indicates that the CFs following FLDR 3 have the minimum local strength of the CFs with a huge difference to the other two FLDR patterns. The minimum local strength of the CFs signifies that the CFs can be more easily controlled in a certain time step $l$, the discrete time scale. This phenomenon further demonstrates the significance of guiding FLDR 1 to be transformed into FLDR 3 through some technical measures or traffic policies.

\section{Summary and Prospect}

The CFs perspective based mesoscopic reliability model of a weighted PTN is the interdisciplinary study between the 


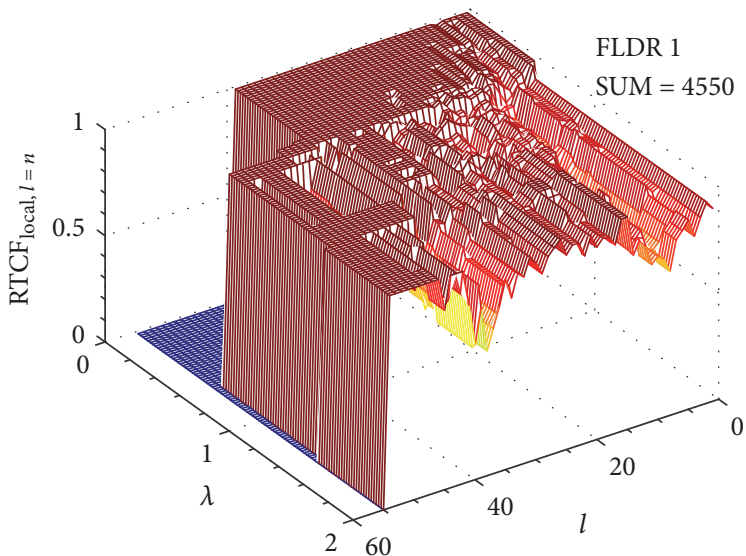

(a)

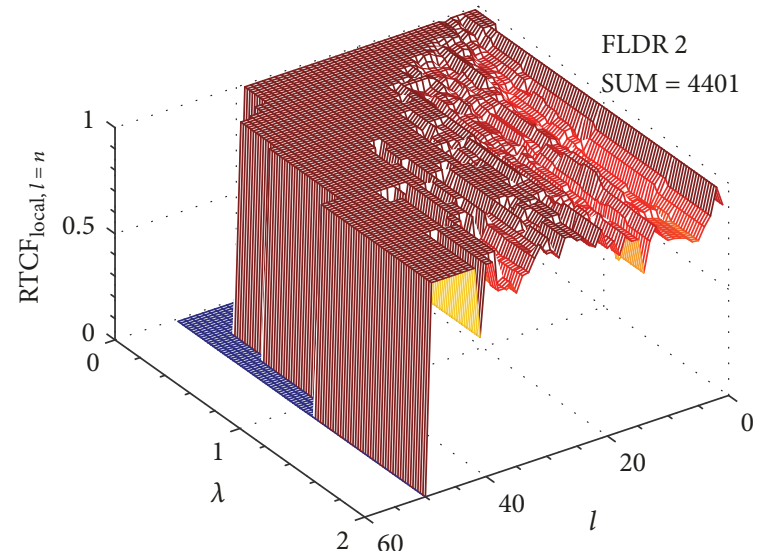

(b)

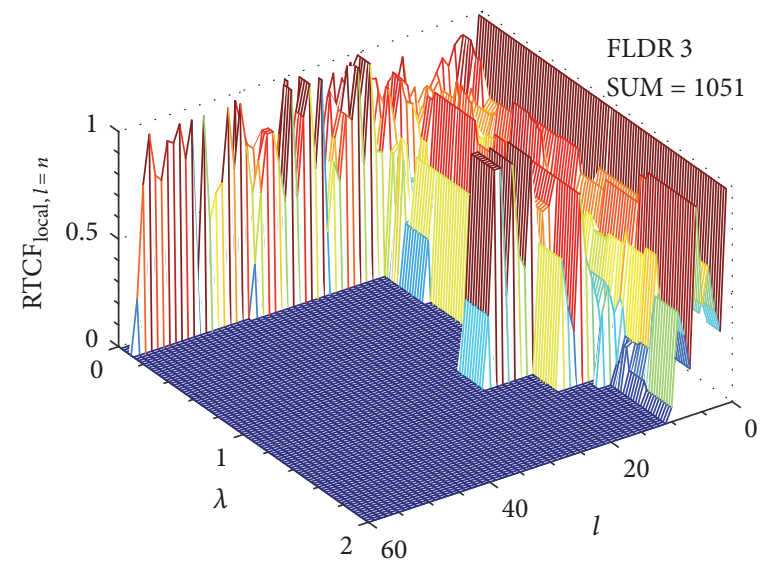

(c)

FIGURE 8: Simulation verification analysis of $\mathrm{RTCF}_{\text {local }, l=n}$ evolution under varying FLDR rules.

transportation engineering and system science, and its ultimate objective is to get an accurate and realistic description of the CFs model which can quantitatively measure the PTN survivability. To overcome the limitation of conventional research methodology that previous studies on the PTN CFs essentially focused on network physical topology although they used the actual networks, this paper establishes a CFs perspective based mesoscopic reliability model of a weighted PTN considering congestion effect and user equilibrium evacuation. First, a modeling method for abstracting weighted PTN and determining its initial passenger flow and bearing capacity is proposed, making the network passenger flow pattern follow the aggregated flow pattern. Second, three basic concepts (time step, congestion effect of a road section, and CFs judging method) for establishing the CFs model are defined to clarify the overall evolution process of CFs. Furthermore, the aggregated passenger flow evacuation that exists in an emergency occurring at a station is considered as a conscious dynamic game process through following the user equilibrium rule. As a result, a novel CFs model that considers congestion effect and user equilibrium evacuation is obtained. Finally, based on the collected data of Jinan's PTN, a case simulation analysis is conducted to verify the adaptability of this model through showing a significantly different dynamics characteristic with the existing FLDR patterns and to provide optimization direction for effectively controlling PTN survivability, that is, guide the transformation among varying FLDR patterns through some technical measures or traffic policies.

Although this paper is based on real PTN data, the parameter settings of the new CFs model are mostly based on empirical assumptions. Additionally, the established weighted PTN is essentially the average passenger flow system of certain period of time, which does not consider the traffic timevarying conditions. In the future, the authors will use the big data platform of PTN to calibrate the parameters and consider the influences of traffic time-varying conditions, so as to provide a more accurate and realistic reliability model for establishing the optimal route layout estimation technology based on reliability optimization.

\section{Conflicts of Interest}

The authors declare that there are no conflicts of interest regarding the publication of this paper. 


\section{Acknowledgments}

This work was supported by the National Natural Science Foundation of China (Grants no. 51478110, no. 51508122, and no. 71471104), the Postgraduate Research \& Practice Innovation Program of Jiangsu Province (no. KYCX17_0144), the Fundamental Research Funds for the Central Universities (no. KYCX17_0144), and the Science and Technology Program of Jiangsu Province (no. BY2016076-05).

\section{References}

[1] J. Tang, F. Liu, W. Zhang, S. Zhang, and Y. Wang, "Exploring dynamic property of traffic flow time series in multi-states based on complex networks: Phase space reconstruction versus visibility graph," Physica A: Statistical Mechanics and its Applications, vol. 450, pp. 635-648, 2016.

[2] G. Gao, H. Sun, J. Wu, and H. Zhao, "Tradable credits scheme and transit investment optimization for a two-mode traffic network," Journal of Advanced Transportation, vol. 50, no. 8, pp. 1616-1629, 2016.

[3] J. Wu, R. Li, R. Ding, T. Li, and H. Sun, "City expansion model based on population diffusion and road growth," Applied Mathematical Modelling: Simulation and Computation for Engineering and Environmental Systems, vol. 21, pp. 40-41, 2016.

[4] L. Han, H. Sun, D. Wang, C. Zhu, and J. Wu, "The combination of continuous network design and route guidance," Computers Operations Research, vol. 73, pp. 92-103, 2016.

[5] L. Zhang, J. Lu, J. Zhou, J. Zhu, Y. Li, and Q. Wan, "Complexities' day-to-day dynamic evolution analysis and prediction for a Didi taxi trip network based on complex network theory," Modern Physics Letters B, vol. 32, no. 09, p. 1850062, 2018.

[6] T. Li, J. Wu, H. Sun, and Z. Gao, "Integrated Co-evolution Model of Land Use and Traffic Network Design," Networks and Spatial Economics, vol. 16, no. 2, pp. 579-603, 2016.

[7] M. Estrada, J. Mensión, J. M. Aymamí, and L. Torres, "Bus control strategies in corridors with signalized intersections," Transportation Research Part C: Emerging Technologies, vol. 71, pp. 500-520, 2016.

[8] S.-X. He, "An anti-bunching strategy to improve bus schedule and headway reliability by making use of the available accurate information," Computers \& Industrial Engineering, vol. 85, article no. 3979, pp. 17-32, 2015.

[9] A. Fonzone, J.-D. Schmöcker, and R. Liu, "A model of bus bunching under reliability-based passenger arrival patterns," Transportation Research Part C: Emerging Technologies, vol. 59, pp. 164-182, 2015.

[10] S. Chakrabarti and G. Giuliano, "Does service reliability determine transit patronage? Insights from the Los Angeles Metro bus system," Transport Policy, vol. 42, pp. 12-20, 2015.

[11] W. Leong, K. Goh, S. Hess, and P. Murphy, "Improving bus service reliability: The Singapore experience," Research in Transportation Economics, vol. 59, pp. 40-49, 2016.

[12] Z. Yu, J. S. Wood, and V. V. Gayah, "Using survival models to estimate bus travel times and associated uncertainties," Transportation Research Part C: Emerging Technologies, vol. 74, pp. 366-382, 2017.

[13] D. J. Watts and S. H. Strogatz, "Collective dynamics of 'smallworld' networks,” Nature, vol. 393, no. 6684, pp. 440-442, 1998.

[14] A.-L. Barabasi and R. Albert, "Emergence of scaling in random networks," Science, vol. 286, no. 5439, pp. 509-512, 1999.
[15] R. Albert, H. Jeong, and A.-L. Barabasi, "Error and attack tolerance of complex networks," Nature, vol. 406, no. 4, pp. 378-382, 2000.

[16] Y. Wang and R. Xiao, "An ant colony based resilience approach to cascading failures in cluster supply network," Physica A: Statistical Mechanics and its Applications, vol. 462, pp. 150-166, 2016.

[17] W. Zhang, W. Pei, and T. Guo, "An efficient method of robustness analysis for power grid under cascading failure," Safety Science, vol. 64, pp. 121-126, 2014.

[18] Y. Koç, M. Warnier, R. E. Kooij, and F. M. T. Brazier, "An entropy-based metric to quantify the robustness of power grids against cascading failures," Safety Science, vol. 59, pp. 126-134, 2013.

[19] F. Xue, E. Bompard, T. Huang, L. Jiang, S. Lu, and H. Zhu, "Interrelation of structure and operational states in cascading failure of overloading lines in power grids," Physica A: Statistical Mechanics and its Applications, vol. 482, pp. 728-740, 2017.

[20] C. Yi, Y. Bao, J. Jiang, and Y. Xue, "Modeling cascading failures with the crisis of trust in social networks," Physica A: Statistical Mechanics and its Applications, vol. 436, pp. 256-271, 2015.

[21] J. J. Wu, H. J. Sun, and Z. Y. Gao, "Cascading failures on weighted urban traffic equilibrium networks," Physica A: Statistical Mechanics and its Applications, vol. 386, no. 1, pp. 407-413, 2007.

[22] J. Sienkiewicz and J. A. Hołyst, "Statistical analysis of 22 public transport networks in Poland," Physical Review E: Statistical, Nonlinear, and Soft Matter Physics, vol. 72, no. 4, Article ID 046127, 2005.

[23] Y.-Z. Chen, N. Li, and D.-R. He, "A study on some urban bus transport networks," Physica A: Statistical Mechanics and its Applications, vol. 376, no. 1-2, pp. 747-754, 2007.

[24] H. Soh, S. Lim, T. Zhang et al., "Weighted complex network analysis of travel routes on the Singapore public transportation system," Physica A: Statistical Mechanics and its Applications, vol. 389, no. 24, pp. 5852-5863, 2010.

[25] J. Wu, Z. Gao, and H. Sun, "Complexity and efficiency of Beijing Transit Network," International Journal of Modern Physics B, vol. 20, no. 15, pp. 21-29, 2006.

[26] B. Berche, C. von Ferber, T. Holovatch, and Y. Holovatch, "Transportation network stability: a case study of city transit," Advances in Complex Systems. A Multidisciplinary Journal, vol. 15, no. suppl. 1, Article ID 1250063, 19 pages, 2012.

[27] Y. Yang, Y. Liu, M. Zhou, F. Li, and C. Sun, "Robustness assessment of urban rail transit based on complex network theory: A case study of the Beijing Subway," Safety Science, vol. 79, pp. 149-162, 2015.

[28] B.-B. Fu, L. Zhang, S.-B. Li, and Y.-X. Li, "Survivability of public transit network based on network structure entropy," International Journal of Modern Physics C, vol. 26, no. 9, Article ID 1550104, 2015.

[29] Z.Zou, Y. Xiao, and J. Gao, "Robustness analysis of urban transit network based on complex networks theory," Kybernetes, vol. 42, no. 3, pp. 383-399, 2013.

[30] L. Zhang, B.-B. Fu, and Y.-X. Li, "Cascading Failure of Urban Weighted Public Transit Network under Single Station Happening Emergency," Procedia Engineering, vol. 137, pp. 259-266, 2016.

[31] T. He, N. Zhu, Z. Hou, and G. Xiong, "A Novel Cascading Failure Model on City Transit Network," in Proceedings of the 2016 6th International Conference on Machinery, Materials, Environment, Biotechnology and Computer, Tianjin, China, June 2016. 
[32] M. Song, X. Weng, S. Yao, and J. Chen, "Research on the importance of the nodes of the cascading failure public transportation network based on complex network theory," Journal of Computational \& Theoretical Nanoscience, vol. 13, no. 8, pp. 5294-5304, 2016.

[33] Y. Sheffi, "Urban transportation networks: equilibrium analysis with mathematical programming methods," Prentice Hall Press, pp. 118-121, 1985.

[34] J. Wang, Y. Li, and Q. Zheng, "Cascading load model in interdependent networks with coupled strength," Physica A: Statistical Mechanics and its Applications, vol. 430, pp. 242-253, 2015.

[35] J. Wang, C. Jiang, and J. Qian, "Robustness of interdependent networks with different link patterns against cascading failures," Physica A: Statistical Mechanics and its Applications, vol. 393, pp. 535-541, 2014.

[36] L. Zhang, B.-B. Fu, and S.-B. Li, "Cascading failures coupled model of interdependent double layered public transit network," International Journal of Modern Physics C, vol. 27, no. 12, Article ID 1650145, 1650145, 18 pages, 2016.

[37] J.-F. Zheng, Z.-Y. Gao, and B.-B. Fu, "Load distribution in congested scale-free networks," International Journal of Modern Physics C, vol. 20, no. 2, pp. 197-207, 2009.

[38] A. Huang, H. M. Zhang, W. Guan, Y. Yang, and G. Zong, "Cascading failures in weighted complex networks of transit systems based on coupled map lattices," Mathematical Problems in Engineering, vol. 2015, Article ID 940795, 2015.

[39] J. G. Wardrop, "Some theoretical aspects of road traffic research," in Proceedings of the Institution of Civil Engineers, vol. 1, pp. 325-362, 1952.

[40] M. J. Beckmann, C. B. McGuire, and C. B. Winsten, Studies in the economics of transportation, Yale University Press, NewHaven, Connecticut, 1956.

[41] M. Frank and P. Wolfe, "An algorithm for quadratic programming," Naval Research Logistics Quarterly, vol. 3, pp. 95-110, 1956.

[42] L. J. LeBlanc, E. K. Morlok, and W. P. Pierskalla, "An efficient approach to solving the road network equilibrium traffic assignment problem," Transportation Research, vol. 9, no. 5, pp. 309318, 1975.

[43] “AutoNai. Traffic analysis report of China's major cities," 2015, http://report.amap.com/download.do.

[44] 2014, Data from http://www.jnbus.com.cn.

[45] L. Zhang, J. Lu, X. Yue, J. Zhou, Y. Li, and Q. Wan, "An auxiliary optimization method for complex public transit route network based on link prediction," Modern Physics Letters B. Condensed Matter Physics, Statistical Physics, Applied Physics, vol. 32, no. 5, 1850066, 22 pages, 2018.

[46] Y. Moreno, R. Pastor-Satorras, A. Vázquez, and A. Vespignani, "Critical load and congestion instabilities in scale-free networks," EPL (Europhysics Letters), vol. 62, no. 2, pp. 292-298, 2003. 


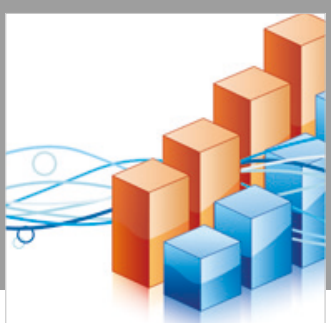

Advances in

Operations Research

\section{-n-m}
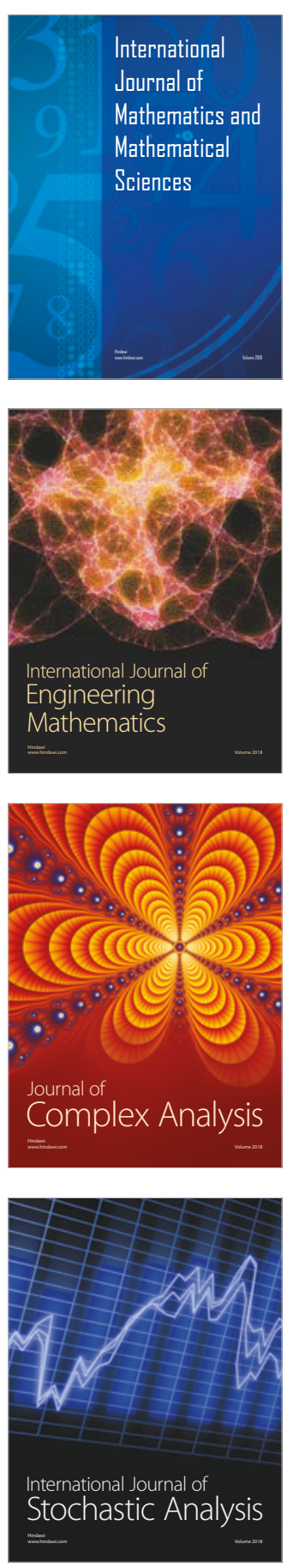
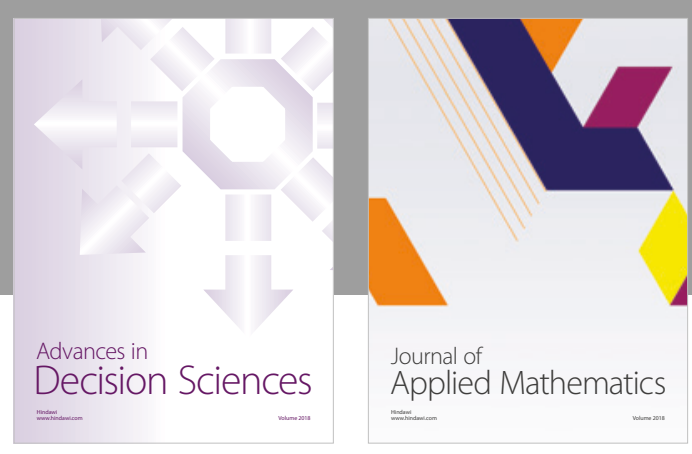

Journal of

Applied Mathematics
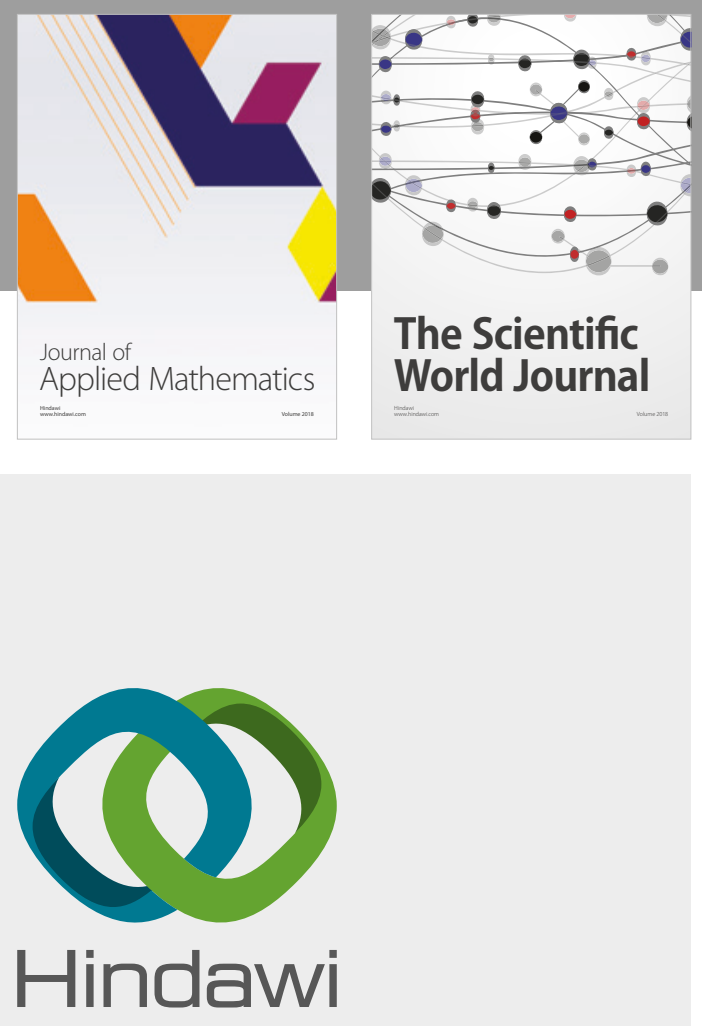

Submit your manuscripts at

www.hindawi.com

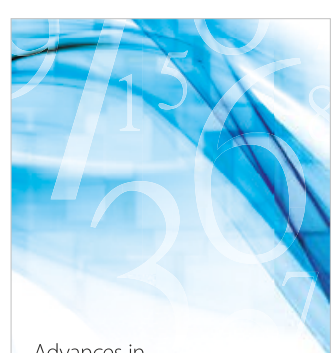

Advances in
Numerical Analysis
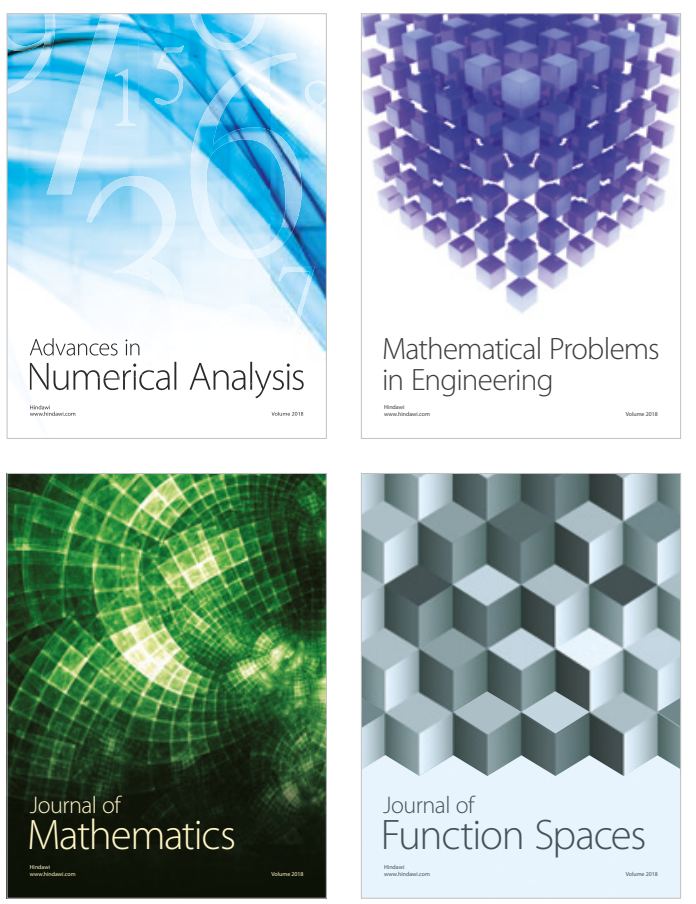

Mathematical Problems in Engineering

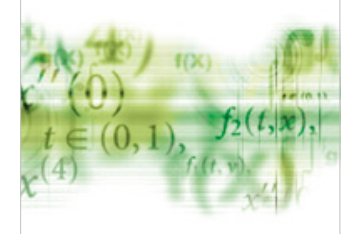

International Journal of

Differential Equations

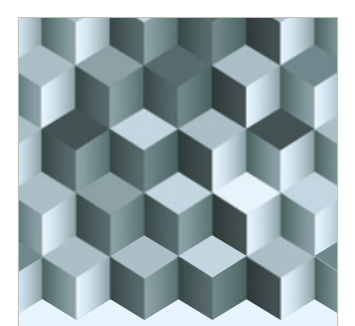

Journal of

Function Spaces

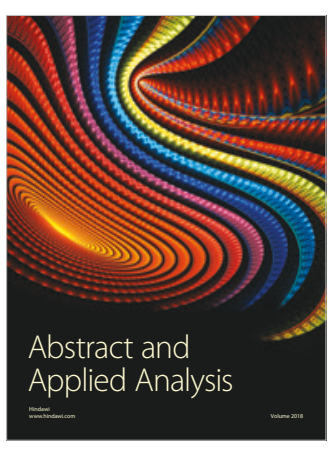

The Scientific

World Journal

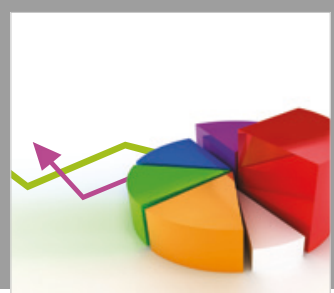

Journal of

Probability and Statistics
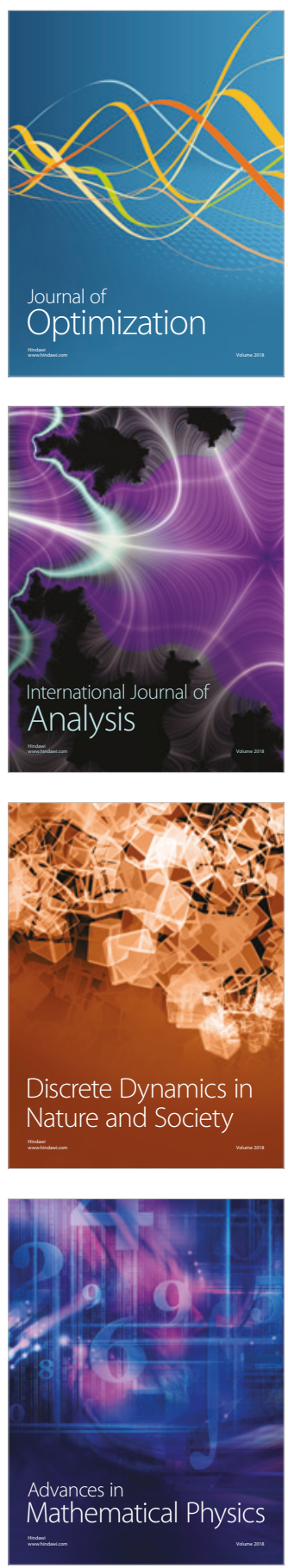